\title{
SMAD3 promotes ELK3 expression following transforming growth factor $\beta$-mediated stimulation of MDA-MB231 cells
}

\author{
JI-HOON PARK and KYUNG-SOON PARK \\ Department of Biomedical Science, College of Life Science, \\ CHA University, Seongnam-si, Gyeonggi-do 463-400, Republic of Korea
}

Received October 1, 2019; Accepted December 19, 2019

DOI: $10.3892 / \mathrm{ol} .2020 .11375$

\begin{abstract}
Transforming growth factor- $\beta$ (TGF $\beta$ ) is a secreted cytokine whose aberrant spatiotemporal expression is related to cancer progression and metastasis. While TGF $\beta$ acts as a tumor suppressor in normal and premalignant stages, TGF $\beta$ functions as a tumor promoter during the malignant phases of tumor progression by prompting cancer cells to undergo epithelial-mesenchymal transition (EMT), which enhances tumor cell invasion and ultimately promotes metastasis to other organs. Extensive studies have been performed to uncover the molecular and cellular mechanisms underlying TGF $\beta$ inducing EMT in cancer cells. Here, we suggested that $E L K 3$, which encodes a protein that orchestrates invasion and metastasis of triple negative breast cancer cells, is a downstream target of TGF $\beta$-SMAD3 in MDA-MB231 cells. ELK3 expression was increased in a time-dependent manner upon TGF $\beta$ treatment. Chemical and molecular inhibition of the TGF $\beta$ receptor blocked the ability of TGF $\beta$ to induce ELK3 expression. Small interfering RNA-mediated suppression analysis revealed that SMAD3 induces TGF $\beta$ signaling to express $E L K 3$. Moreover, the results of the luciferase reporter assay and chromatin immunoprecipitation analysis showed that SMAD3 directly binds to the SMAD-binding element on the promoter of ELK3 to activate gene expression following TGF $\beta$ stimulation. We concluded that ELK3 is a novel downstream target of TGF $\beta$-SMAD3 signaling in aggressive breast cancer cells.
\end{abstract}

Correspondence to: Professor Kyung-Soon Park, Department of Biomedical Science, College of Life Science, CHA University, Pangyo-Ro 335, Bundang-gu, Seongnam-si, Gyeonggi-do 463-400, Republic of Korea

E-mail:kspark@cha.ac.kr

Abbreviations: TGF $\beta$, transforming growth factor- $\beta$; SBE, SMAD binding element; TNBCs, triple negative breast cancer cells; EMT, epithelial-mesenchymal transition; siTGFR1, small interfering RNA targeting to TGF $\beta$ receptor R1; CA-ALK5, constitutively active form of TGF $\beta$ receptor-I

Key words: TGF 3 , SMAD3, ELK3, MDA-MB-231, MCF7, luciferase assay, chromatin immunoprecipitation

\section{Introduction}

Cancer metastasis is the process of cancer cells disseminating from the primary tumor to a distal site through lymphatic tissue and blood vessels. Cancer metastasis is responsible for approximately $90 \%$ of cancer deaths, indicating that it is the primary cause of morbidity and mortality (1). Even though most solid tumors are now manageable or curable by advances in early cancer detection and treatment, cancers spreading beyond the initial primary site are usually highly incurable (2). Lack of understanding of the mechanism underlying the metastatic process has meant that the predominant cancer treatments focus on inhibition of cancer growth with little emphasis on metastasis, meaning that the overall survival of metastatic cancer patients has not been improved significantly.

Transforming growth factor- $\beta$ (TGF $\beta$ ) is one of the master factors of metastasis in that it induces the epithelial-mesenchymal transition (EMT), which is associated with cancer. EMT is the reversible orchestrated transcriptional program in which well-organized, tightly connected epithelial cells transdifferentiate into disorganized and motile mesenchymal cells. TGF- $\beta$ signaling mediated by SMAD or non-SMAD pathways plays a fundamental role in activating the transcriptional network to induce the expression of mesenchymal components and to suppress the expression of epithelial genes $(3,4)$. As a result, epithelial cancer cells undergo dramatic remodeling of the cytoskeleton along with dissolution of tight junctions to acquire mesenchymal features that exhibit a significantly enhanced metastatic dissemination potential into distal organs. This event is induced by the activity of master regulators of EMT, which include SNAIL, SLUG, ZEB1/delta EF1 and ZEB2/SIP1 (5-8).

ELK3 is an ETS domain-containing protein capable of forming a ternary complex with DNA and serum response factor (9). ELK3 is reported to be involved in the migration and invasion of various cancer cells including aggressive basal-like breast cancer cells and liver cancer stem cells $(10,11)$. Previously, we reported that ELK3 suppression impairs the ability of TGF $\beta$ signaling to activate the expression of mesenchymal markers such as Vimentin, Slug and SNAIL in the triple negative breast cancer cells, which suggests that ELK3 is implicated in the TGF $\beta$ signaling pathway to regulate the metastatic process of aggressive cancer cells $(12,13)$. 
In the present study, to extend our understanding of the molecular implication of ELK3 to the TGF $\beta$ signaling pathway in cancer cells, we analyzed the regulatory mechanism of TGF $\beta$ signaling on ELK3 expression. We found that TGF $\beta$ stimulates the transcriptional expression of ELK3 in the representative triple negative breast cancer cell line, MDA-MB231. Furthermore, based on the biochemical and molecular biology study, we demonstrated that TGF $\beta$-mediated phosphorylation of SMAD3 functions as a transcriptional activator of ELK3. Taken together, our data reveal that ELK3 is a direct downstream target of TGF $\beta$-SMAD3 signaling pathway in MDA-MB231 cells.

\section{Materials and methods}

Plasmids, siRNA and primers. Information on the plasmids and siRNAs is summarized in the supplementary Tables SI and SII.

Cell culture and transfection. The triple negative breast cancer cell line MDA-MB231 and the human breast adenocarcinoma cell line MCF7 and the human embryonic kidney $293 \mathrm{~T}$ cells were purchased from American Type Culture Collection (Manassas, VA, USA). These cells were maintained in DMEM (Gibco BRL Life Technologies, Rockville, MD, USA) containing $10 \%(\mathrm{v} / \mathrm{v})$ heat-inactivated fetal bovine serum (Gibco BRL). 293T cells were used for the luciferase assay with the pGL3-ELK3 plasmid. Transient transfection of plasmid DNA or siRNA was performed with Lipofectamine 2000 (Invitrogen, Carlsbad, CA, USA) according to the manufacturer's protocols.

$R N A$ extraction and reverse transcription-quantitative ( $R T-q)$ $P C R$. Total RNA was extracted by manual methods using TRIZol (Invitrogen), and $1 \mu \mathrm{g}$ of cDNA was synthesized using the LeGene Express 1st Strand cDNA Synthesis System (LeGene Biosciences Inc., San Diego, CA, USA) according to the manufacturer's instructions. RT-qPCR was performed using synthetic cDNAs and TOPreal ${ }^{\mathrm{TM}}$ qPCR $2 \mathrm{X}$ PreMIX (Enzynomics, Daejeon, Korea). The expression of the target genes was normalized to that of glyceraldehyde 3-phosphate dehydrogenase (GAPDH). The PCR primers are listed in Table SIII.

Immunoblot analysis. Cells were lysed with RIPA buffer (Cell Signaling Technology, Beverly, MA, USA) and total cell lysates were separated by sodium dodecyl sulfate-polyacrylamide gel electrophoresis (SDS-PAGE) and then transferred to polyvinylidene difluoride membranes (Bio-Rad, Hercules, CA, USA). The membranes were blotted with the indicated primary antibodies at $4^{\circ} \mathrm{C}$ overnight. After washing with TBST, the membranes were incubated for $1 \mathrm{~h}$ at room temperature with secondary antibodies. Immunoreactivity was detected with an ECL kit (Thermo Scientific, Rochester, NY, USA). The antibodies used in this study are summarized in Table SIV.

Luciferase assay. The $293 \mathrm{~T}$ cells were transfected with the indicated plasmids using Lipofectamine 2000 (Invitrogen) according to the manufacturer's protocols. Cells were harvested $48 \mathrm{~h}$ after transfection, and luciferase activity was measured using the Dual-Luciferase Reporter Assay System (Promega) according to the manufacturer's protocols. The values of firefly luciferase were normalized to the respective values of Renilla luciferase.

Chromatin immunoprecipitation. In brief, $37 \%$ formaldehyde was added to the cell culture medium to a final concentration of $1 \%$ and incubated for $15 \mathrm{~min}$ at RT. Glycine was added to a final concentration of $125 \mathrm{mM}$ for $5 \mathrm{~min}$ at $\mathrm{RT}$, and the cells were washed three times with cold PBS. The cells were lysed in $400 \mu \mathrm{l}$ of $1 \mathrm{X}$ cell lysis buffer (Cell Signaling) containing protease/phosphatase inhibitor cocktail (Pierce Biotechnology). After eight rounds of sonication, the lysates were cleared by centrifugation at $13,000 \mathrm{rpm}$ for $15 \mathrm{~min}$ at $4^{\circ} \mathrm{C}$. The supernatants were mixed with $40 \mu \mathrm{l}$ of Dynabead protein $\mathrm{G}$ and $2 \mu \mathrm{g}$ of primary antibodies for $2 \mathrm{~h}$ at RT or overnight at $4^{\circ} \mathrm{C}$. The complexes were washed sequentially with 1X RIPA buffer, 1X RIPA buffer (500 mM NaCl), LiCl buffer and TE buffer twice for $10 \mathrm{~min}$ each. Then, $3 \mu \mathrm{l}$ of $10 \%$ SDS and $5 \mu \mathrm{l}$ of $20 \mathrm{mg} / \mathrm{ml}$ proteinase $\mathrm{K}$ were added to separate the DNA-protein complex. The DNA was purified by the phenol/chloroform extraction method, and then it was used in PCR with primers targeting the ELK3 promoter.

Statistical analysis. Samples were analyzed with Student's t-test or ANOVA with Duncan's multiple range procedure for multiple comparisons. All statistical analyses were performed using GraphPad Prism 5 (GraphPad Prism, USA) or the SigmaPlot 11.2 program (Systat Software, USA). All statistical analyses were performed using GraphPad Prism 5 (GraphPad Prism, USA). The error bars represent the standard errors from three independent experiments, which were each performed using triplicate samples. P-values less than 0.05 were considered statistically significant.

\section{Results}

TGF $\beta$ induces accumulation of ELK3 in the nucleus of MDA-MB231 cells, but not in MCF 7 cells. Cancer cells treated with TGF $\beta$ undergo the EMT process by developing a fibroblast-like morphological appearance and changing epithelial and mesenchymal phenotype marker expression. Unlike MDA-MB231 cells, TGF $\beta$-treated MCF7 cells that display morphological changes of EMT do not show suppression of E-cadherin, a typical epithelial phenotype marker (14). Recently, we reported that ELK3 is highly expressed in TNBC-like MDA-MB231 cells, where it functions as a transcriptional repressor of E-cadherin by collaborating with ZEB1 (15). Therefore, we hypothesized that ELK3 is the missing link that explains the different molecular responses of MDA-MB231 and MCF7 cells when they are treated with TGF $\beta$. We first compared the expression of ELK3 between MDA-MB231 and MCF7 cells following TGF $\beta$ treatment. As expected, TGF $\beta$ stimulated ELK3 expression in MDA-MB231 cells but not in MCF7 cells (Fig. 1A). Consistently, ELK3 protein was also accumulate in the TGF $\beta$-treated MDA-MB231 cells (Fig. 1B). Immunocytochemical analysis and subcellular fractionation assays of the cytosol and nucleus confirmed that ELK3 accumulates in the TGF $\beta$-treated MDA-MB231 cells (Fig. 1C and D). Overall, these data indicate that TGF $\beta$ 
A

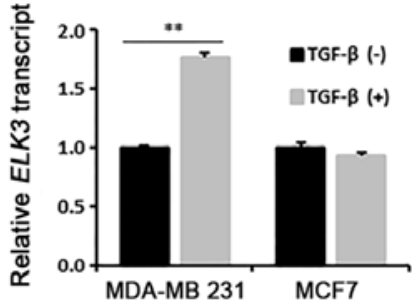

B

Time after TGF $\beta$ treatment $(\mathrm{h})$

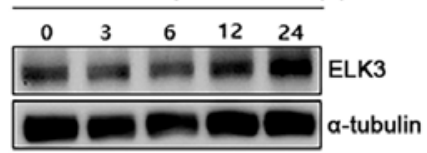
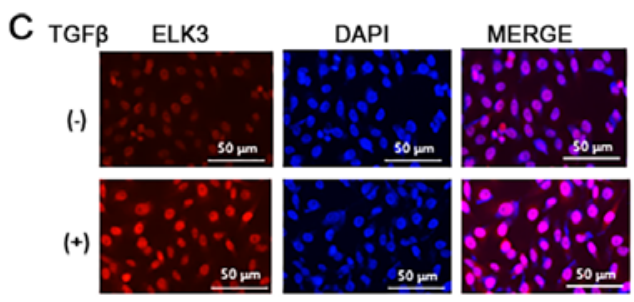

D
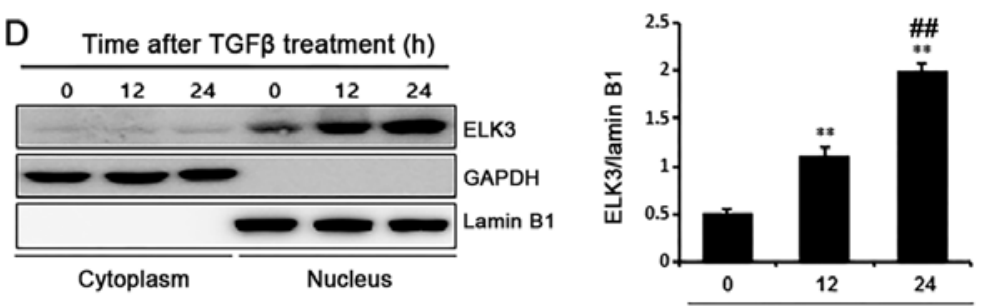

Time after TGF $\beta$ treatment $(\mathrm{h})$

Figure 1. TGF $\beta$ induces accumulation of ELK3 in the nuclei of MDA-MB231 cells. (A) Effect of TGF $\beta$ on the expression of ELK3 in MDA-MB231 and MCF7 cells was compared by RT-qPCR of cancer cells treated with TGF $\beta(5 \mathrm{ng} / \mathrm{ml})$ for $24 \mathrm{~h}$. ${ }^{* *} \mathrm{P}<0.01$. (B) The increase of ELK3 protein (right panel) upon TGF $\beta$ treatment $(5 \mathrm{ng} / \mathrm{ml})$ for the indicated time was analyzed by immunoblot assay. (C) Nuclear accumulation of ELK3 upon TGF $\beta$ treatment was examined in MDA-MB231 cells by immunocytochemical staining. Cells were treated with $5 \mathrm{ng} / \mathrm{ml}$ TGF $\beta$ for $12 \mathrm{~h}$. (D) Nuclear accumulation of ELK3 was examined by immunoblot of nuclear and cytoplasmic fractions from MDA-MB231 cells treated with TGF $\beta$ for the indicated times. A loading control of the cytoplasmic fraction was estimated by GAPDH and a loading control of the nuclear fraction was estimated by Lamin B1 (left panel). For quantitative analysis, the mean density of each band was measured with Multi Gauge V3.0 software, and the band density of EL3 was divided by Lamin B1 to obtain the normalized band intensity (right panel). The error bars represent the standard errors from three independent experiments, which were each performed using triplicate samples. TGF $\beta$, transforming growth factor- $\beta$; RT-qPCR, reverse transcription-quantitative PCR. ${ }^{* *} \mathrm{P}<0.01$ vs. 0 h; ${ }^{\# \#} \mathrm{P}<0.01$ vs. $12 \mathrm{~h}$.
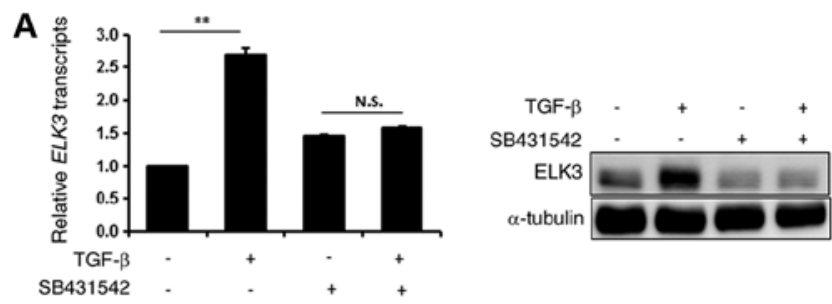

C

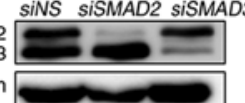

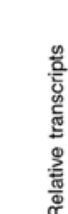

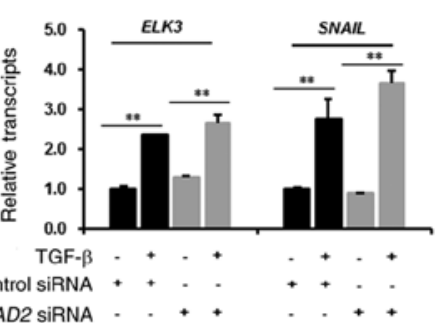

D

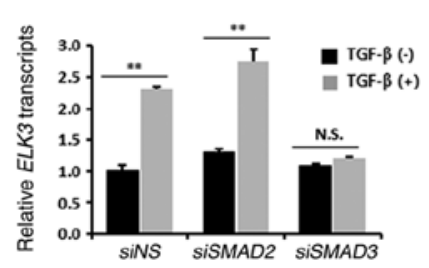

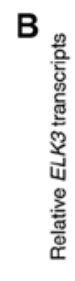
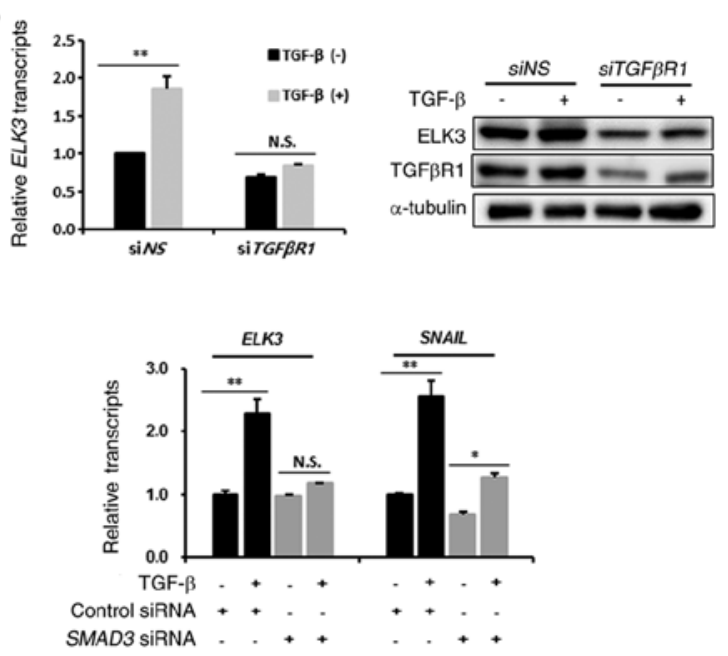

Figure 2. Effect of TGF $\beta$ on $E L K 3$ expression is mediated by SMAD3 but not by SMAD2. (A) Effect of chemical inhibition of the TGF $\beta$ receptor on the TGF $\beta$-mediated activation of ELK3. Cells were pre-incubated with $10 \mu \mathrm{M} \mathrm{SB} 431542$ for $1 \mathrm{~h}$ and then treated with $10 \mathrm{ng} / \mathrm{ml}$ TGF $\beta$ for $24 \mathrm{~h}$. Relative transcripts and protein levels of ELK3 and TGF $\beta$ receptor-I were analyzed by RT-qPCR (left panel) and immunoblot analysis (right panel), respectively. (B) Effect of molecular inhibition of TGF $\beta$ receptor-I on the TGF $\beta$-mediated activation of $E L K 3$ expression. Non-specific or TGF $\beta$ receptor-I targeting siRNAs were transfected into MDA-MB231 cells for $24 \mathrm{~h}$ followed by treatment with $5 \mathrm{ng} / \mathrm{ml}$ of TGF $\beta$ for $24 \mathrm{~h}$. Relative transcripts and protein levels of ELK3 and TGF $\beta$ receptor-I were analyzed by RT-qPCR (left panel) and immunoblot analysis (right panel), respectively. (C) Effect of molecular inhibition of SMAD2 or SMAD3 on the TGF $\beta$-mediated activation of $E L K 3$ expression. Non-specific, SMAD2 or SMAD3 targeting siRNAs were transfected into MDA-MB231 cells for $24 \mathrm{~h}$, which was followed by treatment with $5 \mathrm{ng} / \mathrm{ml}$ of TGF $\beta$ for $24 \mathrm{~h}$. Knockdown effect of SMAD2 and SMAD3 was analyzed by immunoblot analysis (left panel). Relative transcripts and protein levels of ELK3 and SNAIL, which is a target of SMAD3, were analyzed by RT-qPCR (middle and right panel). (D) Effect of molecular inhibition of $S M A D 2$ or $S M A D 3$ on the TGF 3 -treated, time-dependent activation of $E L K 3$ expression. Nonspecific, SMAD2 or SMAD3 targeting siRNAs were transfected into MDA-MB231 cells for $24 \mathrm{~h}$, which was followed by treatment with $5 \mathrm{ng} / \mathrm{ml}$ of TGF $\beta$ for additional $24 \mathrm{~h}$. The expression of $E L K 3$ was quantified by RT-qPCR. The error bars represent the standard errors from three independent experiments, which were each performed using triplicate samples. "P $<0.05$, ${ }^{* *} \mathrm{P}<0.01$. N.S, not significant; TGF $\beta$, transforming growth factor- $\beta$; RT-qPCR, reverse transcription-quantitative PCR; si, small interfering; NS, non-specific. 


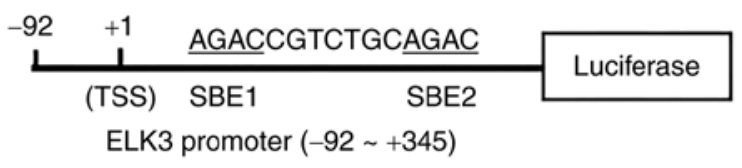

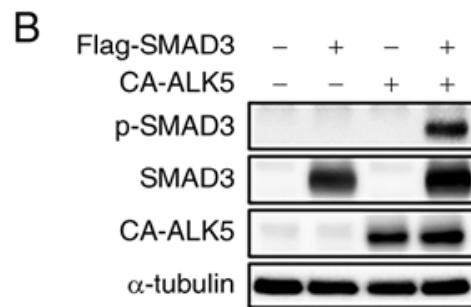

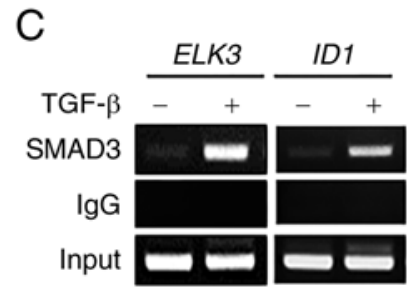

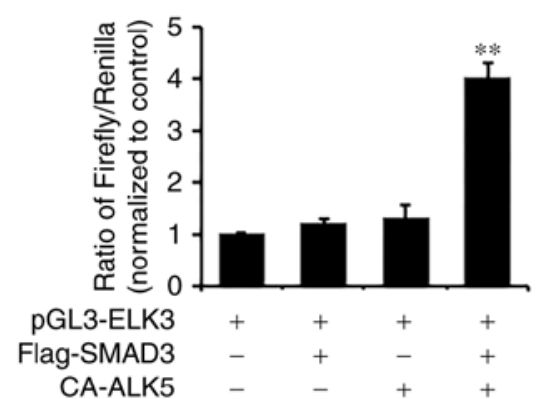

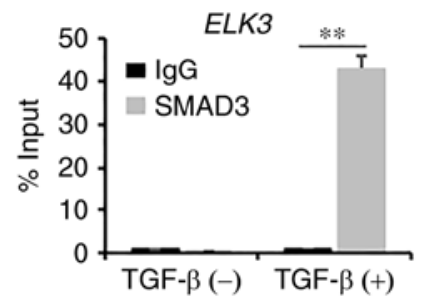

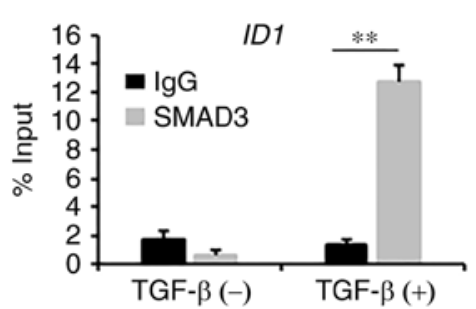

Figure 3. SMAD3 binds to the $E L K 3$ promoter to activate transcription upon TGF $\beta$ treatment. (A) Schematics of luciferase assay promoter construct of $E L K 3$ ( $-92 \mathrm{bp}-+345 \mathrm{bp}$ ) (left panel). The right panel shows the DNA binding motif of SMAD3 in the ELK3 promoter region of human ( $+225 \mathrm{bp}-+257 \mathrm{bp})$ and mouse $(+112$ bp -+144 bp) genomes. (B) Effect of cotransfection of SMAD3 and constitutively active form of the TGF $\beta$ receptor-I (CA-ALK5) on the activity of the ELK3 promoter. Reporter plasmid containing the ELK3 promoter (pGL3-ELK3) was cotransfected into 293T cells with CA-ALK5 and SMAD3 expressing plasmids for $24 \mathrm{hrs}$. The expression of p-SMAD3, SMAD3 and CA-ALK5 was analyzed by immunoblot (left panel), and the activity of the ELK3 promoter was analyzed by luciferase assay (right panel). (C) Chromatin from TGF 3 -treated or nontreated MDA-MB231 cells were immunoprecipitated (ChIP) with antibodies against SMAD3 and IgG. The PCR results for the ELK3 promoter region $(+225 \mathrm{bp}-+257 \mathrm{bp})$ are presented. SMAD3 binding to the ID1 promoter was used as a positive control of the ChIP experiment. The error bars represent the standard errors from three independent experiments, which were each performed using triplicate samples. ${ }^{* *} \mathrm{P}<0.01$. TGF $\beta$, transforming growth factor- $\beta$; CA-ALK5, constitutively active form of TGF $\beta$ receptor-I; $\mathrm{p}$, phosphorylated; ChIP, chromatin immunoprecipitation.

induces transcriptional activation of $E L K 3$ in MDA-MB231 cells but not in MCF7 cells.

TGF $\beta$ activates ELK3 expression via SMAD3. To understand the underlying mechanism of ELK3 activation by TGF $\beta$ treatment, we examined the effect of SB431542, an inhibitor of TGF $\beta$ type I receptor, on the TGF $\beta$-mediated ELK3 expression. As shown in Fig. 2A, pretreatment with SB431542 inhibited mRNA and protein accumulation of ELK3 in the TGF $\beta$-treated MDA-MB231 cells. Consistent with the result of chemical inhibition, transfection of siRNA targeting TGF $\beta$ type I receptor abolished the effect of TGF $\beta$ on the transcriptional activation of ELK3 (Fig. 2B). We next questioned whether $E L K 3$ expression is regulated by SMAD2 or SMAD3. Like SNAIL, which is a downstream target of SMAD3, transfection of an siRNA targeting SMAD3 (siSMAD3) hindered the TGF $\beta$-mediated expression of ELK3, whereas an siRNA targeting SMAD2 (siSMAD2) did not interfere with the TGF $\beta$ effect on ELK3 or SNAIL expression (Fig. 2C). The increase in expression of $E L K 3$ over time in TGF $\beta$-treated MDA-MB231 cells was similar between the control and siSMAD2 transfected MDA-MB231 cells, whereas siSMAD3 transfection abolished the effect of TGF $\beta$ on ELK3 expression (Fig. 2D). Taken together, these results suggest that TGF $\beta$-mediated transcriptional activation of ELK3 is mediated by SMAD3.
SMAD3 binds to the ELK3 promoter to activate the transcription of ELK3 upon TGF $\beta$ treatment. To analyze whether SMAD3 functions as a direct transcriptional activator of $E L K 3$, we examined the sequences of mouse and human $E L K 3$ promoters from $-2,000$ bp to the first exon region. We found that two SMAD3 binding sites (SBE) are conserved at the first exon of the ELK3 promoter in the human and mouse genomes. Therefore, we constructed a luciferase reporter construct containing the promoter region of ELK3 from -92 bp to +345 bp (Fig. 3A). To assess whether SMAD3 functions as a direct transcriptional activator of the ELK3 promoter, the reporter plasmid containing the $E L K 3$ promoter was cotransfected into $293 \mathrm{~T}$ cells with plasmids encoding a constitutively active form of the TGF $\beta$ type I receptor (CA-ALK5) or SMAD3. As shown in Fig. 3B, the ELK3 reporter plasmid is activated only when ectopically expressed SMAD3 is phosphorylated by cotransfection of the CA-ALK5-expressing plasmid in 293T cells. To confirm that SMAD3 directly binds to the $\mathrm{SBE}$ of the ELK3 promoter, we performed chromatin immunoprecipitation (ChIP) analysis with anti-SMAD3 antibody against genomic DNA of MDA-MB231 with or without TGF $\beta$ treatment. Since ID1, an inhibitor of differentiation, is a direct downstream target of SMAD3 (16), it was used as a positive control in the ChIP analysis. Like ID1, the SBE region of the ELK3 promoter was significantly enriched by immunoprecipitation with the anti-SMAD3 antibody upon TGF $\beta$ stimulation 
(Fig. 3C). Taken together, we concluded that SMAD3 activates transcription of $E L K 3$ by directly binding to the SBE region of the $E L K 3$ promoter following TGF $\beta$ treatment.

\section{Discussion}

During cancer development and progression in malignancy, the TGF $\beta$ signaling pathway acts as a tumor promotor by driving EMT, which induces tumor cell migration, invasion and ultimately metastasis to distant organs. ELK3 is constitutively activated in basal triple negative breast cancer cells (TNBCs) and functions as a master regulator of cancer metastasis $(10,12)$. Previously, we suggested that the TGF $\beta$ signaling pathway is interconnected with ELK3 activity, based on the fact that ELK3 knockdown in TNBCs induces collapse of TGF $\beta$ signaling (12). In this study, we demonstrated that ELK3 is transcriptionally activated by TGF $\beta$ treatment in TNBCs. Pharmacological and molecular analysis revealed that ELK3 is a direct downstream target of SMAD3. In addition, TGF $\beta$ induced migration was decreased in ELK3 knockdowned MDA-MB231 cells (data not shown).

There are numerous reports that the TGF $\beta$ signaling pathway is strictly regulated by a finely tuned system of negative and positive feedback loops. The expression of SMAD7, a representative inhibitory SMAD, is stimulated by TGF $\beta$ treatment and forms a complex with E3 ubiquitin ligase to degrade the TGF $\beta$ receptor, which results in the SMAD pathway inhibiting hyper activation of TGF $\beta$ signaling (17). During late stages of colorectal cancer, TGF $\beta$ activates miR-1269a expression targeting SMAD7, hence forming a positive feedback loop to promote metastasis (18). Since TGF $\beta$ signaling is impaired by ELK3 suppression and ELK3 expression is increased by TGF $\beta$ treatment, we suggest that TGF $\beta$ and ELK3 might form a positive autofeedback loop to promote the EMT process.

Numerous studies have shown that inhibition of EMT is considered an appropriate approach towards the prevention of metastasis of cancer. Since TGF $\beta$ functions as an inducer of EMT, blocking the TGF $\beta$ pathway is considered a promising strategy to inhibit EMT in cancer cells; cytotoxic drugs such as paclitaxel, which targets TGF $\beta$ receptor kinase, have been used to target the metastatic potential of breast cancer cells to colonize the lung (19). In line with this concept, ELK3 can be a prominent therapeutic target to prevent TGF $\beta$-mediated metastasis of cancer cells. The potential value of ELK3 as a target of anticancer drug development is supported by the fact that TNBCs with reduced ELK 3 activity completely lost their metastatic characteristics (12). It was shown that small molecule based inhibition of Ras/ERK-mediated ELK3 activity results in the inhibition of prostate cancer progression and metastasis in mice (20). It would be interesting to investigate whether simultaneous inhibition of the TGF $\beta$ pathway and ELK 3 activity produces clinically effective therapeutic outcomes.

In summary, we suggest that ELK3 is a novel downstream target of the TGF $\beta$-SMAD3 signaling pathway and that it performs a major role in directing the metastasis of cancer. TGF- $\beta 1$ is preferentially expressed at the advancing tumor edges, where it promotes malignant progression and metastasis (21-23). To strengthen our findings, follow-up immunohistochemical studies are needed to demonstrate the accumulation of ELK3 at the site of excessive TGF $\beta$ expression on invasive tumors.

\section{Acknowledgements}

The authors would like to thank Dr Seong-Jin Kim (Seoul National University) for providing the expression plasmids containing FLAG-SMAD3 and constitutively active ALK5 (CA-ALK5).

\section{Funding}

The present study was supported by The Ministry of Education, Science, and Technology (NRF-2019R1A2C1003581) and by Basic Science Research Program through The National Research Foundation of Korea (NRF) funded by The Ministry of Education (grant no. 2019R1A6A1A03032888).

\section{Availability of data and materials}

The datasets used and/or analyzed during the present study are available from the corresponding author on reasonable request.

\section{Authors' contributions}

JHP designed the experiment and performed all experiments. KSP made substantial contributions to the analysis and interpretation of data. KSP has also been involved in drafting the manuscript and revising it critically for important intellectual content. JHP agreed to the final version of the manuscript.

\section{Ethics approval and consent to participate}

Not applicable.

\section{Patient consent for publication}

Not applicable.

\section{Competing interests}

The authors declare that they have no competing interests.

\section{References}

1. Seyfried TN and Huysentruyt LC: On the origin of cancer metastasis. Crit Rev Oncog 18: 43-73, 2013.

2. Wells A, Grahovac J, Wheeler S, Ma B and Lauffenburger D: Targeting tumor cell motility as a strategy against invasion and metastasis. Trends Pharmacol Sci 34: 283-289, 2013.

3. Xu J, Lamouille S and Derynck R: TGF-beta-induced epithelial to mesenchymal transition. Cell Res 19: 156-172, 2009.

4. Thiery JP and Sleeman JP: Complex networks orchestrate epithelial-mesenchymal transitions. Nat Rev Mol Cell Biol 7: 131-142, 2006.

5. Cano A, Pérez-Moreno MA, Rodrigo I, Locascio A, Blanco MJ, del Barrio MG, Portillo F and Nieto MA: The transcription factor snail controls epithelial-mesenchymal transitions by repressing E-cadherin expression. Nat Cell Biol 2: 76-83, 2000.

6. Savagner P, Yamada KM and Thiery JP: The zinc-finger protein slug causes desmosome dissociation, an initial and necessary step for growth factor-induced epithelial-mesenchymal transition. J Cell Biol 137: 1403-1419, 1997. 
7. Eger A, Aigner K, Sonderegger S, Dampier B, Oehler S, Schreiber M, Berx G, Cano A, Beug H and Foisner R: DeltaEF1 is a transcriptional repressor of E-cadherin and regulates epithelial plasticity in breast cancer cells. Oncogene 24: 2375-2385, 2005.

8. Comijn J, Berx G, Vermassen P, Verschueren K, van Grunsven L, Bruyneel E, Mareel M, Huylebroeck D and van Roy F: The two-handed $E$ box binding zinc finger protein SIP1 downregulates E-cadherin and induces invasion. Mol Cell 7: 1267-1278, 2001.

9. Buchwalter G, Gross C and Wasylyk B: Ets ternary complex transcription factors. Gene 324: 1-14, 2004

10. Heo SH, Lee JY, Yang KM and Park KS: ELK3 expression correlates with cell migration, invasion, and membrane type 1-matrix metalloproteinase expression in MDA-MB-231 breast cancer cells. Gene Expr 16: 197-203, 2015.

11. Lee JH, Hur W, Hong SW, Kim JH, Kim SM, Lee EB and Yoon SK: ELK3 promotes the migration and invasion of liver cancer stem cells by targeting HIF-1 $\alpha$. Oncol Rep 37: 813-822, 2017.

12. Kong SY, Kim KS, Kim J, Kim MK, Lee KH, Lee JY, Oh N, Park JI, Park JH, Heo SH, et al: The ELK3-GATA3 axis orchestrates invasion and metastasis of breast cancer cells in vitro and in vivo. Oncotarget 7: 65137-65146, 2016.

13. Kim KS, Kim J, Oh N, Kim MY and Park KS: ELK3-GATA3 axis modulates MDA-MB-231 metastasis by regulating cell-cell adhesion-related genes. Biochem Biophys Res Commun 498 509-515, 2018.

14. Lv ZD, Kong B, Li JG, Qu HL, Wang XG, Cao WH, Liu XY, Wang Y, Yang ZC, Xu HM and Wang HB: Transforming growth factor-beta 1 enhances the invasiveness of breast cancer cells by inducing a Smad2-dependent epithelial-to-mesenchymal transition. Oncol Rep 29: 219-225, 2013.

15. Cho HJ, Oh N, Park JH, Kim KS, Kim HK, Lee E, Hwang S, Kim SJ and Park KS: ZEB1 collaborates with ELK3 to repress E-cadherin expression in triple negative breast cancer cells. Mol Cancer Res 17: 2257-2266, 2019.
16. Liang YY, Brunicardi FC and Lin X: Smad 3 mediates immediate early induction of Id1 by TGF-beta. Cell Res 19: 140-148, 2009.

17. Kavsak P, Rasmussen RK, Causing CG, Bonni S, Zhu H, Thomsen GH and Wrana JL: Smad7 binds to Smurf2 to form an E3 ubiquitin ligase that targets the TGF beta receptor for degradation. Mol Cell 6: 1365-1375, 2000.

18. Bu P, Wang L, Chen KY, Rakhilin N, Sun J, Closa A, Tung KL, King S, Kristine Varanko A, Xu Y, et al: miR-1269 promotes metastasis and forms a positive feedback loop with TGF- $\beta$. Nat Commun 6: 6879, 2015 .

19. Park SY, Kim MJ, Park SA, Kim JS, Min KN, Kim DK, Lim W, Nam JS and Sheen YY: Combinatorial TGF- $\beta$ attenuation with paclitaxel inhibits the epithelial-to-mesenchymal transition and breast cancer stem-like cells. Oncotarget 6: 37526-37543, 2015.

20. Semenchenko K, Wasylyk C, Cheung H, Tourrette Y, Maas P, Schalken JA, van der Pluijm G and Wasylyk B: XRP44X, an inhibitor of Ras/Erk activation of the transcription factor Elk3, inhibits tumour growth and metastasis in mice. PLoS One 11: e0159531, 2016.

21. Pickup M, Novitskiy S and Moses HL: The roles of TGF $\beta$ in the tumour microenvironment. Nat Rev Cancer 13: 788-799, 2013.

22. Dalal BI, Keown PA and Greenberg AH: Immunocytochemical localization of secreted transforming growth factor-beta 1 to the advancing edges of primary tumors and to lymph node metastases of human mammary carcinoma. Am J Pathol 143: 381-389, 1993.

23. Steiner MS, Zhou ZZ, Tonb DC and Barrack ER: Expression of transforming growth factor-beta 1 in prostate cancer. Endocrinology 135: 2240-2247, 1994.

(i) $\Theta$ This work is licensed under a Creative Commons Attribution-NonCommercial-NoDerivatives 4.0 International (CC BY-NC-ND 4.0) License. 